\title{
Minimal thinness with respect to the Schrödinger operator and its applications on singular Schrödinger-type boundary value problems
}

\section{Bo Meng ${ }^{1 *}$}

"Correspondence:

bo.mengust@gmail.com

${ }^{1}$ College of Mathematics and

Systems Science, Shandong

University of Science and

Technology, Qingdao, P.R. China

\begin{abstract}
The application of the new criteria for minimally thin sets with respect to the Schrödinger operator to an approximate solution of singular Schrödinger-type boundary value problems are discussed in this study. The method is based on approximating functions and their derivatives by using the natural and weakened total energies. This study shows that the new criteria are very effective and powerful tools in solving such problems. At the end of the paper, we are also concerned with the boundary behaviors of solutions for a kind of quasilinear Schrödinger equation.
\end{abstract}

Keywords: Schrödinger-type boundary value problem; Boundary behavior; Schrödinger equation

\section{Introduction}

In this paper, we further consider the following Schrödinger problem (see [1]):

$$
i z_{t}=-\Delta z+W(x) z-a(x) h\left(|z|^{2}\right) z-k \Delta l\left(|z|^{2}\right) l^{\prime}\left(|z|^{2}\right) z,
$$

where $x \in \mathbb{R}^{n}, z: \mathbb{R} \times \mathbb{R}^{n} \rightarrow \mathbb{C}, a, W: \mathbb{R}^{n} \rightarrow \mathbb{R}$ is a given potential, $k$ is real constant, and $l$ and $h$ are real functions. The above quasilinear equations have been accepted as models of several physical phenomena corresponding to various types of $l$; we refer to [2] and the references given therein for physical applications of these problems. Specifically, we would like to mention that the superfluid film equation in plasma physics has this structure for $l(s)=s$ (see e.g. [3, 4]), while in the case $l(s)=(1+s)^{1 / 2},(1)$ models the self-channeling of a high-power ultrashort laser in matter (see e.g. $[5,6]$ ).

The standing waves solutions of (1); that is, solutions of the type $z(t, x)=\exp (-i E t) u(x)$ where $E \in \mathbb{R}$ and $u>0$ is a real function. Inserting $z$ into (1), with $l(s)=s$ and $l(s)=(1+$ $\left.s^{2}\right)^{1 / 2}$, turns, respectively, the following equations (see e.g. [7]):

$$
\begin{aligned}
& \left.-\Delta u+V_{\infty} u-k \Delta\left(u^{2}\right)\right) u=a(x) h(u), \\
& -\Delta u+V_{\infty} u-k \Delta\left(\left(1+u^{2}\right)^{1 / 2}\right) \frac{u}{\left(1+u^{2}\right)^{1 / 2}}=a(x) h(u),
\end{aligned}
$$

where $x \in \mathbb{R}^{n}$ and $V_{\infty}=W-E$.

(c) The Author(s) 2019. This article is distributed under the terms of the Creative Commons Attribution 4.0 International License (http://creativecommons.org/licenses/by/4.0/), which permits unrestricted use, distribution, and reproduction in any medium, provided you give appropriate credit to the original author(s) and the source, provide a link to the Creative Commons license, and indicate if changes were made. 
It is well known that an unknown Borel probability measure on $W=S \times T$ controls the sampling process, where $T=\mathbb{R}$ and $S$ is a compact metric space in $\mathbb{R}^{n}$. As in [8], the exact weak solutions of $(1)$ can be defined by $g_{\varrho}(s)=\int_{T} y d \varrho(t \mid s)$, where $\varrho(\cdot \mid s)$ is the conditional probability measure induced by $\varrho$ on $T$ given $s \in S$.

To our knowledge, the criteria for minimally thin sets with respect to the Schrödinger operator (1) was introduced for the first time in the context of the stationary Schrödinger equations in $[9,10]$. In 2018, Jiang, Zhang and $\mathrm{Li}$ (see [11]) further improved this complex method and applied to study meromorphic solutions for the linear differential equations with analytic coefficients and obtain some applications. Recently, Zhang (see [12, 13]) defined a new type of minimal thinness with respect to the stationary Schrödinger operator, established new criteria for it and applied the result to study growth properties at infinity of the maximum modulus with respect to the Schrödinger operator.

In this paper, we will continue to apply new criteria for solutions for a kind of quasilinear Schrödinger equations. Although we are motivated here by [9-13], there were substantial difficulties to adapt the above approach to the present situation. Let $\mathfrak{H}_{E}$ be the completion of the linear span of the set of functions $\left\{E_{s}:=E(s, \cdot): s \in S\right\}$ equipped with (see $\left.[8,14]\right)$

$$
\left\langle\sum_{i=1}^{n} \xi_{i} E_{s_{i}}, \sum_{l=1}^{m} \phi_{j} E_{t_{j}}\right\rangle_{E}:=\sum_{i=1}^{n} \sum_{l=1}^{m} \xi_{i} \phi_{j} E\left(s_{i}, t_{j}\right) .
$$

Let $s \in S$ and $g \in \mathfrak{H}_{E}$. Define (see [15, Remark 2.3])

$$
g(s)=\left\langle g, E_{s}\right\rangle_{E}
$$

It follows from (2) that (see [16])

$$
\|g\|_{\infty} \leq \kappa\|g\|_{E}
$$

where

$$
\kappa:=\sup _{t, s \in S}|E(s, t)|<\infty .
$$

Define (see [17])

$$
\begin{aligned}
& g_{\mathbf{w}, \chi}(s)=g_{\mathbf{w}, \zeta, \chi, s}(s)=\left.g_{\mathbf{w}, \zeta, \chi, s}(u)\right|_{u=s}, \\
& g_{\mathbf{w}, \zeta, \chi, s}:=\arg \min _{f \in \mathfrak{H}_{E}}\left\{\frac{1}{m} \sum_{i=1}^{m} \Phi\left(\frac{s}{\zeta}, \frac{s_{i}}{\zeta}\right)\left(t_{i}-g\left(s_{i}\right)\right)^{2}+\chi\|g\|_{E}^{2}\right\},
\end{aligned}
$$

where

$$
\begin{aligned}
& \Phi(s, t) \leq 1, \quad \forall s, t \in \mathbb{R}^{n}, \\
& \Phi(s, t) \geq c_{q}, \quad \forall|s-t| \leq 1 .
\end{aligned}
$$

Scheme (4) yields (see $[18,19])$

$$
g_{\mathbf{w}, \zeta}(s)=g_{\mathbf{w}, \zeta, \zeta, s}(s)=\left.g_{\mathbf{w}, \zeta, \zeta, s}(u)\right|_{u=s},
$$




$$
g_{\mathbf{w}, \zeta, \zeta, s}=\arg \min _{f \in \mathfrak{H}_{E, \mathbf{w}}}\left\{\frac{1}{m} \sum_{i=1}^{m} \Psi\left(\frac{s}{\zeta}, \frac{s_{i}}{\zeta}\right)\left(g\left(s_{i}\right)-t_{i}\right)^{2}+\varsigma \sum_{i=1}^{m}\left|\xi_{i}\right|^{q}\right\}
$$

and

$$
\mathfrak{H}_{E, \mathbf{w}}=\left\{g(s)=\sum_{i=1}^{m} \xi_{i} E\left(s, s_{i}\right): \xi=\left(\xi_{1}, \ldots, \xi_{m}\right) \in \mathbb{R}^{m}, m \in \mathbb{N}\right\} .
$$

In order to study the boundary behaviors of $g_{\mathbf{w}, \varsigma}$, we derive

$$
\left\|g_{\mathbf{w}, \varsigma}-g_{\varrho}\right\|_{\varrho S}
$$

with (see [20-23] for more details)

$$
\|g(\cdot)\|_{\varrho S}:=\left(\int_{S}|g(\cdot)|^{2} d \varrho_{S}\right)^{\frac{1}{2}}
$$

The remainder of this paper is organized as follows. In Sect. 2, we will provide the main results. In Sect. 3, some basic but important estimates and properties are summarized. The proofs of main results will be given in Sect. 4. Section 5 contains the conclusions of the paper.

\section{Main results}

The integral operator $L_{E}: L_{\varrho S}^{2}(S) \rightarrow L_{\varrho S}^{2}(S)$ is defined by

$$
\left(L_{E} g\right)(s)=\int_{S} E(s, t) g(t) d \varrho_{S}(t)
$$

Let $\left\{\mu_{i}\right\}$ be the eigenvalues of $L_{E}$ and $\left\{e_{i}\right\}$ be the corresponding eigenfunctions. Then we define

$$
L_{E}^{r}(g)=\sum_{i=1}^{\infty} \mu_{i}^{r}\left\langle g, e_{i}\right\rangle_{L_{\varrho_{S}}^{2}} e_{i}
$$

for $g \in L_{\varrho S}^{2}(S)$. We assume that $g_{\varrho}$ satisfies $L_{E}^{-r} g_{\varrho} \in L_{\varrho S}^{2}$, where $r$ is a positive constant depending on the size of the initial data in a suitable norm.

Let $c_{p}(0<p<2)$ be a positive constant. Define (see [24])

$$
\log \mathfrak{N}_{2}\left(B_{1}, \epsilon\right) \leq c_{p} \epsilon^{-p}
$$

where

$$
B_{1}=\left\{f \in \mathfrak{H}_{E, \mathbf{w}}:\|g\|_{E} \leq 1\right\} .
$$

Now we are in a position to obtain the existence of solutions for the problem (1). 
Theorem 1 Suppose $L_{E}^{-r} g_{\varrho} \in L_{\varrho S}^{2}$ with $r>0$, (7) with $0<p<2$. Then there exist solutions for the problem (1), which can be defined by

$$
\mathfrak{H}(\mathbf{w}, \chi, \varsigma)=\int_{S}\left(\mathfrak{E}_{\mathbf{w}, s}\left(\gamma_{M}\left(g_{\mathbf{w}, \zeta, \zeta, s}\right)\right)+\varsigma \Omega_{\mathbf{w}}\left(g_{\mathbf{w}, \zeta, \zeta, s}\right)\right) d \varrho_{S}(s)
$$

and

$$
\mathfrak{H}(\mathbf{w}, \chi, \varsigma) \leq \frac{m \varsigma M^{2}}{(m \chi)^{q}}
$$

For the further application of Theorem 1, we have the following result. Similar results for solutions of the stationary Schrödinger equations, we refer the reader to the papers $($ see $[13,25])$.

Proposition 1 Let $L_{E}^{-r} g_{\varrho} \in L_{\varrho S}^{2}$, where $r>0$. Then

$$
\mathfrak{D}(\chi) \leq C_{1} \chi^{\min \{2 r, 1\}} .
$$

It follows from Theorem 1 that we can decompose solutions for the problem (1) into two parts, $\mathfrak{H}_{1}(\mathbf{w}, \varsigma)+\mathfrak{H}_{2}(\mathbf{w}, \chi)$, where

$$
\int_{S}\left\{\mathfrak{E}_{s}\left(\gamma_{M}\left(g_{\mathbf{w}, \zeta, \zeta, s}\right)\right)-\mathfrak{E}_{s}\left(g_{\varrho}\right)-\mathfrak{E}_{\mathbf{w}, s}\left(\gamma_{M}\left(g_{\mathbf{w}, \zeta, \zeta, s}\right)\right)+\mathfrak{E}_{\mathbf{w}, s}\left(g_{\varrho}\right)\right\} d \varrho_{S}(s)
$$

and

$$
\int_{S}\left\{\mathfrak{E}_{\mathbf{w}, s}\left(g_{\chi}\right)-\mathfrak{E}_{\mathbf{w}, s}\left(g_{\varrho}\right)-\mathfrak{E}_{s}\left(g_{\chi}\right)+\mathfrak{E}_{s}\left(g_{\varrho}\right)\right\} d \varrho_{S}(s)
$$

Finally, we further study the boundary behaviors for solutions for the problem (1).

Theorem 2 Let the assumptions of Theorem 1 hold. Then

$$
\mathfrak{H}_{2}(\mathbf{w}, \chi) \leq \frac{\mathfrak{D}(\chi)}{2}+\frac{7\left(3 M+\kappa \sqrt{\frac{\mathfrak{D}(\chi)}{\chi}}\right)^{2} \log (2 / \delta)}{3 m}
$$

where $0<\delta<1$.

Theorem 3 Let the assumptions of Theorem 1 hold. Then

$$
\begin{aligned}
\mathfrak{H}_{1}(\mathbf{w}, \varsigma) \leq & \frac{1}{2} \int_{S}\left\{\mathfrak{E}_{s}\left(\gamma_{M}\left(g_{\mathbf{w}, \zeta, \zeta, S}\right)\right)-\mathfrak{E}_{s}\left(g_{\varrho}\right)\right\} d \varrho_{S}(s) \\
& +\frac{176 M^{2}}{m} \log \left(\frac{2}{\delta}\right)+C_{p, M} R_{\zeta}^{\frac{2 p}{2+p}} m^{-\frac{2}{2+p}},
\end{aligned}
$$

where $0<\delta<1$ and

$$
R_{\varsigma}=\kappa m^{1-\frac{1}{q}}\left(\frac{M^{2}}{\varsigma}\right)^{\frac{1}{q}}
$$




\section{Lemmas}

Some basic but important estimates are needed in this section. The following lemma indicates that the natural and weakened total energies are conserved in time.

Lemma 1 We have the following estimates:

$$
\begin{aligned}
\mathfrak{E}_{\tau, g}(t) & =\mathfrak{E}_{\tau, g}(0), \quad \forall t \in[0, \tau], \\
\widetilde{E}_{\tau, g}(t)=\widetilde{E}_{\tau, g}(0), & \forall t \in[0, \tau] .
\end{aligned}
$$

Proof Multiplying the first equation by $g_{\varrho}^{\prime}$, we obtain

$$
\left\langle g_{\varrho}^{\prime \prime}(t)-\partial_{g}^{2} g_{\varrho}(t)+\delta g_{\chi}(t), g_{\varrho}^{\prime}(t)\right\rangle_{\mathbb{R}^{N}, g}=0 .
$$

It follows that

$$
\left\langle g_{\varrho}^{\prime \prime}(t), g_{\varrho}^{\prime}(t)\right\rangle_{\mathbb{R}^{N, g}}+\left\langle\left(-\partial_{g}^{2}\right)^{1 / 2} g_{\varrho}(t),\left(-\partial_{g}^{2}\right)^{1 / 2} g_{\varrho}^{\prime}(t)\right\rangle_{\mathbb{R}^{N, g}}+\delta\left\langle g_{\chi}(t), g_{\varrho}^{\prime}(t)\right\rangle_{\mathbb{R}^{N}, g}=0
$$

Therefore

$$
\frac{d}{d t} \gamma_{M}\left(g_{\varrho} ; t\right)+\delta\left\langle g_{\chi}(t), g_{\varrho}^{\prime}(t)\right\rangle_{\mathbb{R}^{N}, g}=0
$$

which leads to

$$
\frac{d}{d t} \gamma_{M}\left(g_{\chi} ; t\right)+\delta\left\langle g_{\varrho}(t), g_{\chi}^{\prime}(t)\right\rangle_{\mathbb{R}^{N}, g}=0
$$

Adding (13) and (14), we can write

$$
\frac{d}{d t} \mathfrak{E}_{\tau, g}(t)=0
$$

which is equivalent to (11).

By taking the sum of the resulting two identities we obtain

$$
\frac{d}{d t} \widetilde{\mathfrak{E}}_{g}\left(g_{\varrho} ; t\right)+\frac{d}{d t} \widetilde{\mathfrak{E}}_{g}\left(g_{\chi} ; t\right)+\delta\left\langle g_{\chi}(t),\left(-\partial_{g}^{2}\right)^{-1} g_{\varrho}^{\prime}(t)\right\rangle_{\mathbb{R}^{N}, g}+\delta\left\langle g_{\varrho}(t),\left(-\partial_{g}^{2}\right)^{-1} g_{\chi}^{\prime}(t)\right\rangle_{\mathbb{R}^{N}, g}=0,
$$

using the symmetry of the matrix $\left(-\partial_{g}^{2}\right)^{-1}$ we obtain

$$
\frac{d}{d t} \widetilde{\mathfrak{E}}_{\tau, g}(t)=0
$$

From Lemma 1, we deduce the following result.

Lemma 2 Let $0 \leq \delta \leq \frac{\delta_{0}}{3}$. Then

$$
\int_{S}\left(\gamma_{M}\left(g_{\varrho} ; t\right)+\widetilde{\mathfrak{E}}_{g}\left(g_{\chi} ; t\right)\right) d t \geq \frac{C \tau}{2}\left(\widetilde{\mathfrak{E}}_{g}\left(g_{\varrho} ; 0\right)+\widetilde{\mathfrak{E}}_{g}\left(g_{\chi} ; 0\right)\right)
$$

for a positive constant $C \tau$ depending only on $\tau$. 
Proof We recall

$$
\gamma_{M}\left(g_{\varrho} ; t\right)=\frac{1}{2}\left\|g_{\varrho}^{\prime}(t)\right\|_{\mathbb{R}^{N}, g}^{2}+\frac{1}{2}\left\|\left(-\partial_{g}^{2}\right)^{1 / 2} g_{\varrho}(t)\right\|_{\mathbb{R}^{N}, g}^{2},
$$

and we can write

$$
\gamma_{M}\left(g_{\varrho} ; t\right) \geq \frac{\delta_{0}}{2}\left\|\left(-\partial_{g}^{2}\right)^{-1 / 2} g_{\varrho}^{\prime}(t)\right\|_{\mathbb{R}^{N}, g}^{2}+\frac{\delta_{0}}{2}\left\|g_{\varrho}(t)\right\|_{\mathbb{R}^{N}, g}^{2}=\delta_{0} \widetilde{\mathfrak{E}}_{g}\left(g_{\varrho} ; t\right) .
$$

It follows from Lemma 1 that

$$
\int_{S}\left(\gamma_{M}\left(g_{\varrho} ; t\right)+\widetilde{\mathfrak{E}}_{g}\left(g_{\chi} ; t\right)\right) d t \geq C \int_{S}\left(\widetilde{\mathfrak{E}}_{g}\left(g_{\varrho} ; t\right)+\widetilde{\mathfrak{E}}_{g}\left(g_{\chi} ; t\right)\right) d t .
$$

On the other hand

$$
\left|\widetilde{\mathfrak{E}}_{\tau, g}(t)-\left(\widetilde{\mathfrak{E}}_{g}\left(g_{\varrho} ; t\right)+\widetilde{\mathfrak{E}}_{g}\left(g_{\chi} ; t\right)\right)\right|=\left|\delta\left\langle\left(-\partial_{g}^{2}\right)^{-1} g_{\varrho}(t), g_{\chi}(t)\right\rangle_{\mathbb{R}^{N}, g}\right|,
$$

and thanks to Lemma 1 and [26, Theorem 2.1], one has

$$
\left|\widetilde{\mathfrak{E}}_{\tau, g}(t)-\left(\widetilde{\mathfrak{E}}_{g}\left(g_{\varrho} ; t\right)+\widetilde{\mathfrak{E}}_{g}\left(g_{\chi} ; t\right)\right)\right| \leq \frac{\delta}{\delta_{0}}\left(\widetilde{\mathfrak{E}}_{g}\left(g_{\varrho} ; t\right)+\widetilde{\mathfrak{E}}_{g}\left(g_{\chi} ; t\right)\right) .
$$

Hence

$$
\widetilde{\mathfrak{E}}_{g}\left(g_{\varrho} ; t\right)+\widetilde{\mathfrak{E}}_{g}\left(g_{\chi} ; t\right) \geq \frac{\delta_{0}}{\delta_{0}+\delta} \widetilde{\mathfrak{E}}_{\tau, g}(t) .
$$

Integrating this last inequality over $t \in[0, \tau]$ and using the fact that the energy $\widetilde{\mathfrak{E}}_{\tau, g}(t)$ is conservative, we deduce that

$$
\int_{S}\left(\widetilde{\mathfrak{E}}_{g}\left(g_{\varrho} ; t\right)+\widetilde{\mathfrak{E}}_{g}\left(g_{\chi} ; t\right)\right) d t \geq \frac{\delta_{0} \tau}{\delta_{0}+\delta} \widetilde{\mathfrak{E}}_{\tau, g}(0) .
$$

Moreover, thanks to inequality (17), we have

$$
\widetilde{\mathfrak{E}}_{\tau, g}(0) \geq \frac{\delta_{0}-\delta}{\delta_{0}}\left(\widetilde{\mathfrak{E}}_{g}\left(g_{\varrho} ; 0\right)+\widetilde{\mathfrak{E}}_{g}\left(g_{\chi} ; 0\right)\right),
$$

and inserting this last equation into (18) yields

$$
\int_{S}\left(\widetilde{\mathfrak{E}}_{g}\left(g_{\varrho} ; t\right)+\widetilde{\mathfrak{E}}_{g}\left(g_{\chi} ; t\right)\right) d t \geq \frac{\delta_{0}-\delta}{\delta_{0}+\delta} \tau\left(\widetilde{\mathfrak{E}}_{g}\left(g_{\varrho} ; 0\right)+\widetilde{\mathfrak{E}}_{g}\left(g_{\chi} ; 0\right)\right)
$$

However, since

$$
\frac{\delta_{0}-\delta}{\delta_{0}+\delta} \geq \frac{1}{2}
$$

for all $\delta \leq \frac{\delta_{0}}{3}$, we deduce from (19) that

$$
\int_{S}\left(\widetilde{\mathfrak{E}}_{g}\left(g_{\varrho} ; t\right)+\widetilde{\mathfrak{E}}_{g}\left(g_{\chi} ; t\right)\right) d t \geq \frac{\tau}{2}\left(\widetilde{\mathfrak{E}}_{g}\left(g_{\varrho} ; 0\right)+\widetilde{\mathfrak{E}}_{g}\left(g_{\chi} ; 0\right)\right) .
$$

Inserting this inequality into (16), the desired estimate (15) is obtained. 
We complete this subsection with the following lemma.

Lemma 3 We have

$$
\begin{aligned}
\int_{S} \widetilde{\mathfrak{E}}_{g}\left(g_{\chi} ; t\right) d t \leq & \frac{C}{\delta\left(\sqrt{\delta_{0}}-\delta\right)}\left(\gamma_{M}\left(g_{\varrho} ; 0\right)+\widetilde{\mathfrak{E}}_{g}\left(g_{\chi} ; 0\right)\right) \\
& +\frac{C}{\left(\sqrt{\delta_{0}}-\delta\right)^{2}} \int_{S} \gamma_{M}\left(g_{\varrho} ; t\right) d t, \\
\int_{S}\left\|g_{\chi}(t)\right\|_{\mathbb{R}^{N}, g}^{2} d t \leq & \frac{C}{\delta\left(\sqrt{\delta_{0}}-\delta\right)}\left(\gamma_{M}\left(g_{\varrho} ; 0\right)+\widetilde{\mathfrak{E}}_{g}\left(g_{\chi} ; 0\right)\right) \\
& +\frac{C}{\left(\sqrt{\delta_{0}}-\delta\right)^{2}} \int_{S} \gamma_{M}\left(g_{\varrho} ; t\right) d t, \\
\widetilde{\mathfrak{E}}_{g}\left(g_{\chi} ; \tau\right)+\widetilde{\mathfrak{E}}_{g}\left(g_{\chi} ; 0\right) \leq & \frac{C}{\sqrt{\delta_{0}}-\delta}\left(\gamma_{M}\left(g_{\varrho} ; 0\right)+\widetilde{\mathfrak{E}}_{g}\left(g_{\chi} ; 0\right)\right) \\
& +\frac{C \delta}{\left(\sqrt{\delta_{0}}-\delta\right)^{2}} \int_{S} \gamma_{M}\left(g_{\varrho} ; t\right) d t,
\end{aligned}
$$

where $0 \leq \delta \leq \min \left(\delta_{0}, \sqrt{\delta_{0}}\right)$.

Proof First, we recall the following estimates:

$$
\begin{aligned}
\int_{S}\left\|g_{\chi}(t)\right\|_{\mathbb{R}^{N}, g}^{2} d t \leq & \frac{C}{\delta\left(\sqrt{\delta_{0}}-\delta\right)}\left(\gamma_{M}\left(g_{\varrho} ; 0\right)+\widetilde{\mathfrak{E}}_{g}\left(g_{\chi} ; 0\right)\right) \\
& +\frac{C}{\left(\sqrt{\delta_{0}}-\delta\right)^{2}} \int_{S}\left(\left\|g_{\varrho}(t)\right\|_{\mathbb{R}^{N}, g}^{2}+\left\|g_{\varrho}^{\prime}(t)\right\|_{\mathbb{R}^{N}, g}^{2}\right) d t, \\
\int_{S}\left\|\left(-\partial_{g}^{2}\right)^{-1 / 2} g_{\chi}^{\prime}(t)\right\|_{\mathbb{R}^{N}, g}^{2} d t \leq & \frac{C}{\delta\left(\sqrt{\delta_{0}}-\delta\right)}\left(\gamma_{M}\left(g_{\varrho} ; 0\right)+\widetilde{\mathfrak{E}}_{g}\left(g_{\chi} ; 0\right)\right) \\
& \quad \frac{C}{\left(\sqrt{\delta_{0}}-\delta\right)^{2}} \int_{S}\left(\left\|g_{\varrho}(t)\right\|_{\mathbb{R}^{N}, g}^{2}+\left\|g_{\varrho}^{\prime}(t)\right\|_{\mathbb{R}^{N}, g}^{2}\right) d t,
\end{aligned}
$$

from the proof of Lemma 2.

Taking the sum of these two inequalities, we obtain

$$
\begin{aligned}
\int_{S} \widetilde{\mathfrak{E}}_{g}\left(g_{\chi} ; t\right) d t \leq & \frac{C}{\delta\left(\sqrt{\delta_{0}}-\delta\right)}\left(\gamma_{M}\left(g_{\varrho} ; 0\right)+\widetilde{\mathfrak{E}}_{g}\left(g_{\chi} ; 0\right)\right) \\
& +\frac{C}{\left(\sqrt{\delta_{0}}-\delta\right)^{2}} \int_{S}\left(\left\|g_{\varrho}(t)\right\|_{\mathbb{R}^{N}, g}^{2}+\left\|g_{\varrho}^{\prime}(t)\right\|_{\mathbb{R}^{N}, g}^{2}\right) d t .
\end{aligned}
$$

And thanks to Lemma 2, we improve (24) as follows:

$$
\begin{aligned}
\int_{S} \widetilde{\mathfrak{E}}_{g}\left(g_{\chi} ; t\right) d t \leq & \frac{C}{\delta\left(\sqrt{\delta_{0}}-\delta\right)}\left(\gamma_{M}\left(g_{\varrho} ; 0\right)+\widetilde{\mathfrak{E}}_{g}\left(g_{\chi} ; 0\right)\right) \\
& +\frac{C}{\left(\sqrt{\delta_{0}}-\delta\right)^{2}} \int_{S} \gamma_{M}\left(g_{\varrho} ; t\right) d t
\end{aligned}
$$

which proves the inequality (20). 
The other estimates (21) and (22), are obtained easily from equations (23), (24) and the relation

$$
\int_{S}\left(\left\|g_{\varrho}(t)\right\|_{\mathbb{R}^{N, g}}^{2}+\left\|g_{\varrho}^{\prime}(t)\right\|_{\mathbb{R}^{N}, g}^{2}\right) d t \leq \max \left(\frac{1}{\delta_{0}}, 1\right) \int_{S} \gamma_{M}\left(g_{\varrho} ; t\right) d t .
$$

\section{Proofs of main results}

Now we derive the learning rates.

Proof of Theorem 1 Let $\mathbf{y}=\left(t_{1}, t_{2}, t_{3}, \ldots, t_{m}\right)^{\tau}, K[\mathbf{s}]=\left(E\left(s_{i}, s_{j}\right)\right)_{i, j=1}^{m}$ and $\mathbf{a}^{\mathbf{w}}=\left(a_{1}^{\mathbf{w}}, \ldots, a_{m}^{\mathbf{w}}\right)$ be the coefficient of $g_{\mathbf{w}, \varsigma}$. It follows from the representation theorem (see $\left.[27,28]\right)$ that

$$
a_{i}^{\mathbf{w}}=\frac{1}{\chi m} \Psi\left(\frac{s}{\zeta}, \frac{s_{i}}{\zeta}\right)\left(t_{i}-g_{\mathbf{w}, \zeta, \chi, s}\left(s_{i}\right)\right)
$$

for $i=1,2, \ldots, m$.

By the Hölder inequality, we have

$$
\begin{aligned}
\sum_{i=1}^{m}\left|a_{i}^{\mathbf{w}}\right|^{q} & =\frac{1}{(\chi m)^{q}} \sum_{i=1}^{m}\left|\Psi\left(\frac{s}{\zeta}, \frac{s_{i}}{\zeta}\right)\left(t_{i}-g_{\mathbf{w}, \zeta, \chi, s}\left(s_{i}\right)\right)\right|^{q} \\
\leq & \frac{1}{(\chi m)^{q}}\left(\sum_{i=1}^{m} \Psi\left(\frac{s}{\zeta}, \frac{s_{i}}{\zeta}\right)^{\frac{1}{2-q}}\right)^{1-\frac{q}{2}} \\
& \times\left(\sum_{i=1}^{m}\left(\frac{s}{\zeta}, \frac{s_{i}}{\zeta}\right)\left(t_{i}-g_{\mathbf{w}, \zeta, \chi, s}\left(s_{i}\right)\right)^{2}\right)^{\frac{q}{2}}
\end{aligned}
$$

It follows that

$$
\sum_{i=1}^{m}\left|a_{i}^{\mathbf{w}}\right|^{q} \leq \frac{m}{(\chi m)^{q}}\left(\mathfrak{E}_{\mathbf{w}, s}\left(g_{\mathbf{w}, \zeta, \chi, s}\right)\right)^{\frac{q}{2}}
$$

from (5).

Thus

$$
\begin{aligned}
\mathfrak{E}_{\mathbf{w}, s} & \left(\gamma_{M}\left(g_{\mathbf{w}, \zeta, \zeta, s}\right)\right)+\varsigma \Omega_{\mathbf{w}}\left(g_{\mathbf{w}, \zeta, \zeta, s}\right) \\
\leq & \mathfrak{E}_{\mathbf{w}, s}\left(g_{\mathbf{w}, \zeta, \zeta, s}\right)+\varsigma \Omega_{\mathbf{w}}\left(g_{\mathbf{w}, \zeta, \zeta, s}\right) \\
\leq & \mathfrak{E}_{\mathbf{w}, s}\left(g_{\mathbf{w}, \zeta, \chi, s}\right)+\varsigma \Omega_{\mathbf{w}}\left(g_{\mathbf{w}, \zeta, \chi, s}\right) \\
\leq & \mathfrak{E}_{\mathbf{w}, s}\left(g_{\mathbf{w}, \zeta, \chi, s}\right)+\frac{m \zeta}{(\chi m)^{q}}\left(\mathfrak{E}_{\mathbf{w}, s}\left(g_{\mathbf{w}, \zeta, \chi, s}\right)\right)^{\frac{q}{2}} \\
\leq & \mathfrak{E}_{\mathbf{w}, s}\left(g_{\mathbf{w}, \zeta, \chi, s}\right)+\chi\left\|g_{\mathbf{w}, \zeta, \chi, s}\right\|_{E}^{2} \\
& +\frac{m \zeta}{(\chi m)^{q}}\left(\mathfrak{E}_{\mathbf{w}, s}\left(g_{\mathbf{w}, \zeta, \chi, s}\right)+\chi\left\|g_{\mathbf{w}, \zeta, \chi, s}\right\|_{E}^{2}\right)^{\frac{q}{2}} .
\end{aligned}
$$

Since

$$
\mathfrak{E}_{\mathbf{w}, s}\left(g_{\mathbf{w}, \zeta, \chi, s}\right)+\chi\left\|g_{\mathbf{w}, \zeta, \chi, s}\right\|_{E}^{2} \leq \mathfrak{E}_{\mathbf{w}, s}(0)+\chi\|0\|_{E}^{2},
$$


we get

$$
\begin{aligned}
& \mathfrak{E}_{\mathbf{w}, s}\left(\gamma_{M}\left(g_{\mathbf{w}, \zeta, \zeta, s}\right)\right)+\varsigma \Omega_{\mathbf{w}}\left(g_{\mathbf{w}, \zeta, \zeta, s}\right) \\
& \leq \mathfrak{E}_{\mathbf{w}, s}\left(g_{\mathbf{w}, \zeta, \chi, s}\right)+\chi\left\|g_{\mathbf{w}, \zeta, \chi, s}\right\|_{E}^{2}+\frac{m \zeta M^{2}}{(\chi m)^{q}} .
\end{aligned}
$$

This yields our desired estimation.

Proof of Theorem 2 Let

$$
h(u, t)=\int_{S} \Psi\left(\frac{s}{\zeta}, \frac{u}{\zeta}\right)\left[\left(t-g_{\chi}(u)\right)^{2}-\left(t-g_{\varrho}(u)\right)^{2}\right] d \varrho_{S}(s)
$$

for any $z=(u, t) \in Z$. Then

$$
\begin{aligned}
& \int_{Z} h d \varrho=\int_{S}\left\{\mathfrak{E}_{s}\left(g_{\chi}\right)-\mathfrak{E}_{s}\left(g_{\varrho}\right)\right\} d \varrho_{S}(s) \\
& \frac{1}{m} \sum_{i=1}^{m} h\left(w_{i}\right)=\int_{S}\left\{\mathfrak{E}_{\mathbf{w}, s}\left(g_{\chi}\right)-\mathfrak{E}_{\mathbf{w}, s}\left(g_{\varrho}\right)\right\} d \varrho_{S}(s) .
\end{aligned}
$$

By (3) we have

$$
\left\|g_{\chi}\right\|_{\infty} \leq \kappa\left\|g_{\chi}\right\|_{E} \leq \kappa \sqrt{\frac{\mathfrak{D}(\chi)}{\chi}} .
$$

Combining with (5), we have

$$
\begin{aligned}
|h(u, t)| & \leq\left(\left\|g_{\chi}\right\|_{\infty}+M\right)\left(3 M+\left\|g_{\chi}\right\|_{\infty}\right) \\
& \leq\left(3 M+\kappa \sqrt{\frac{\mathfrak{D}(\chi)}{\chi}}\right)^{2}:=B_{\chi} .
\end{aligned}
$$

Therefore

$$
\left\|h(u, t)-\int_{Z} h d \varrho\right\|_{\infty} \leq 2 B_{\chi}
$$

and

$$
\begin{aligned}
\zeta^{2}(h) \leq & \int_{Z} h^{2} d \varrho \\
= & \int_{Z}\left(\int_{S} \Psi\left(\frac{s}{\zeta}, \frac{u}{\zeta}\right) d \varrho_{S}(s)\right)^{2}\left(g_{\chi}(u)-g_{\varrho}(u)\right)^{2} \\
& \times\left(g_{\chi}(u)+g_{\varrho}(u)-2 y\right)^{2} d \varrho(u, t) \\
\leq & \left(3 M+\left\|g_{\chi}\right\|_{\infty}\right)^{2}\left\|g_{\chi}-g_{\varrho}\right\|_{\varrho S}^{2} \\
\leq & B_{\chi} \mathfrak{D}(\chi) .
\end{aligned}
$$


By Lemma 1,

$$
\frac{1}{m} \sum_{i=1}^{m} h\left(w_{i}\right)-\int_{Z} h d \varrho \leq \frac{\mathfrak{D}(\chi)}{2}+\frac{7 B_{\chi} \log (2 / \delta)}{3 m} .
$$

Proof of Theorem 3 Consider the set of functions

$$
\mathfrak{G}_{R}=\left\{h(u, t)=\int_{S} \Psi\left(\frac{s}{\zeta}, \frac{u}{\zeta}\right)\left(\left(t-\gamma_{M}(g)(u)\right)^{2}-\left(t-g_{\varrho}(u)\right)^{2}\right) d \varrho_{S}(s): f \in B_{R}\right\} .
$$

We have

$$
\begin{aligned}
|h(u, t)| & \leq \int_{S} \Psi\left(\frac{s}{\zeta}, \frac{u}{\zeta}\right)\left|\left(\gamma_{M}(g)(u)-g_{\varrho}(u)\right) \times\left(\gamma_{M}(g)(u)+g_{\varrho}(u)-2 y\right)\right| d \varrho_{S}(s) \\
& \leq 8 M^{2}
\end{aligned}
$$

from (5), which yields

$$
\begin{aligned}
|h(u, t)|^{2} & =\left|\int_{S} \Psi\left(\frac{s}{\zeta}, \frac{u}{\zeta}\right)\left(\gamma_{M}(g)(u)-g_{\varrho}(u)\right) \times\left(\gamma_{M}(g)(u)+g_{\varrho}(u)-2 y\right) d \varrho_{S}(s)\right|^{2} \\
& \leq 16 M^{2} \int_{S} \Psi\left(\frac{s}{\zeta}, \frac{u}{\zeta}\right)\left(\gamma_{M}(g)(u)-g_{\varrho}(u)\right)^{2} d \varrho_{S}(s) \int_{S} \Psi\left(\frac{s}{\zeta}, \frac{u}{\zeta}\right) d \varrho_{S}(s) .
\end{aligned}
$$

So

$$
\mathfrak{E}\left(h^{2}\right) \leq 16 M^{2} \int_{S}\left(\int_{S} \Psi\left(\frac{s}{\zeta}, \frac{u}{\zeta}\right)\left(\gamma_{M}(g)(u)-g_{\varrho}(u)\right)^{2} d \varrho_{S}(u)\right) d \varrho_{S}(s)
$$

It has been proved in $[13,29]$ that

$$
\begin{aligned}
\int_{S} \Psi\left(\frac{s}{\zeta}, \frac{u}{\zeta}\right)\left(g(u)-g_{\varrho}(u)\right)^{2} d \varrho_{S}(u) \\
=\int_{Z} \Psi\left(\frac{s}{\zeta}, \frac{u}{\zeta}\right)\left[(g(u)-t)^{2}-\left(g_{\varrho}(u)-t\right)^{2}\right] d \varrho(u, t),
\end{aligned}
$$

which implies that

$$
\begin{aligned}
\mathfrak{E}\left(h^{2}\right) \leq & 16 M^{2} \int_{S}\left(\int _ { Z } \Psi ( \frac { s } { \zeta } , \frac { u } { \zeta } ) \left[\left(\gamma_{M}(g)(u)-t\right)^{2}\right.\right. \\
& \left.\left.-\left(g_{\varrho}(u)-t\right)^{2}\right] d \varrho(u, t)\right) d \varrho_{S}(s) \\
= & 16 M^{2} \int_{Z}\left(\int _ { S } \Psi ( \frac { s } { \zeta } , \frac { u } { \zeta } ) \left[\left(\gamma_{M}(g)(u)-t\right)^{2}\right.\right. \\
& \left.\left.-\left(g_{\varrho}(u)-t\right)^{2}\right] d \varrho_{S}(s)\right) d \varrho(u, t) \\
= & 16 M^{2} \mathfrak{E}(h) .
\end{aligned}
$$


Then we get

$$
\begin{aligned}
& \left|h_{1}(u, t)-h_{2}(u, t)\right| \\
& =\left|\int_{S} \Psi\left(\frac{s}{\zeta}, \frac{u}{\zeta}\right)\left(\left(\gamma_{M}\left(g_{1}\right)(u)-t\right)^{2}-\left(\gamma_{M}\left(g_{2}\right)(u)-t\right)^{2}\right) d \varrho_{S}(s)\right| \\
& \left.\leq \mid \int_{S} \Psi\left(\frac{s}{\zeta}, \frac{u}{\zeta}\right)\left(\gamma_{M}\left(g_{1}\right)(u)\right)-\gamma_{M}\left(g_{2}\right)(u)\right) \\
& \quad \times\left(\gamma_{M}\left(g_{1}\right)(u)+\gamma_{M}\left(g_{2}\right)(u)-2 t\right) d \varrho_{S}(s) \mid \\
& \leq 4 M\left|g_{1}(u)-g_{2}(u)\right|
\end{aligned}
$$

for any $h_{1}, h_{2} \in \mathfrak{G}_{R}$, which yields

$$
\mathfrak{N}_{2}\left(\mathfrak{G}_{R}, \varepsilon\right) \leq \mathfrak{N}_{2}\left(B_{R}, \frac{\varepsilon}{4 M}\right)=\mathfrak{N}_{2}\left(B_{1}, \frac{\varepsilon}{4 M R}\right) .
$$

It follows from the capacity condition (7) that

$$
\log \mathfrak{N}_{2}\left(\mathfrak{G}_{R}, \epsilon\right) \leq c_{p}(4 M)^{p} R^{p} \epsilon^{-p} .
$$

By applying Lemma 2 to $g$ with $Q=8 M^{2}$ we have

$$
\mathfrak{E} g-\frac{1}{m} \sum_{i=1}^{m} h\left(w_{i}\right) \leq \frac{\mathfrak{E g} g}{2}+\frac{176 M^{2}}{m} \log \left(\frac{2}{\delta}\right)+C_{p, M} R^{\frac{2 p}{2+p}} m^{-\frac{2}{2+p}}
$$

for any $0<\delta<1$, where

$$
C_{p, M}=c_{p}^{\prime}(4 M)^{\frac{4}{2+p}} c_{p}^{\frac{2}{2+p}}
$$

Moreover, we take $f=g_{\mathbf{w}, \zeta, 5, s}$ and derive the following bound of $g_{\mathbf{w}, \zeta, 5, s}$ by using the same method in [9, Lemma 3] and (5):

$$
\left\|g_{\mathbf{w}, \varsigma}\right\|_{E} \leq \kappa m^{1-\frac{1}{q}}\left(\frac{M^{2}}{\varsigma}\right)^{\frac{1}{q}} .
$$

If we take

$$
R=R_{\varsigma}=\kappa m^{1-\frac{1}{q}}\left(\frac{M^{2}}{\varsigma}\right)^{\frac{1}{q}},
$$

then we can complete the proof of Theorem 3.

\section{Conclusion}

The application of the new criteria for minimally thin sets with respect to the Schrödinger operator to an approximate solution of singular Schrödinger-type boundary value problems were discussed in this study. The method was based on approximating functions and 
their derivatives by using the natural and weakened total energies. This study showed that the new criteria were very effective and powerful tools in solving such problems. At the end of the paper, we were also concerned with the boundary behaviors of solutions for a kind of quasilinear Schrödinger equation.

Acknowledgements

Not applicable.

\section{Funding}

This work was supported by the Post-Doctoral Applied Research Projects of Qingdao (no. 2015122) and the Scientific Research Foundation of Shandong University of Science and Technology for Recruited Talents (no. 2014RCJJ032).

\section{Abbreviations \\ Not applicable.}

\section{Availability of data and materials}

Not applicable.

Ethics approval and consent to participate

Not applicable.

\section{Competing interests}

The author declares that he has no competing interests.

\section{Consent for publication}

Not applicable.

\section{Authors' contributions}

The author read and approved the final manuscript.

\section{Publisher's Note}

Springer Nature remains neutral with regard to jurisdictional claims in published maps and institutional affiliations.

Received: 27 December 2018 Accepted: 8 May 2019 Published online: 15 May 2019

\section{References}

1. Cottle, R.: Nonlinear programs with positively bounded jacobians. Ph.D. Dissertation, Department of Mathematics, University of California, Berkeley (1964)

2. Glowinski, R., Lions, J., Trémolières, R.: Numerical Analysis of Variational Inequalities. North-Holland, Amsterdam (1981)

3. Cui, Y., Ma, W., Sun, Q., Su, X.: New uniqueness results for boundary value problem of fractional differential equation. Nonlinear Anal., Model. Control 23(1), 31-39 (2018)

4. Cui, Y., Ma, W., Wang, X., Su, X.: Uniqueness theorem of differential system with coupled integral boundary conditions. Electron. J. Qual. Theory Differ. Equ. 9, 1 (2018)

5. Zou, Y., He, G.: A fixed point theorem for systems of nonlinear operator equations and applications to (p1,p2)-Laplacian system. Mediterr. J. Math. 15(2), 74 (2018)

6. Zhang, X., Liu, L., Wu, Y., Cui, Y.: The existence and nonexistence of entire large solutions for a quasilinear Schrödinger elliptic system by dual approach. J. Math. Anal. Appl. 464(2), 1089-1106 (2018)

7. Lions, J., Stampaccia, G.: Variational inequalities. Commun. Pure Appl. Math. 20, 493-512 (1967)

8. Bremermann, J.: Distributions, Complex Variables, and Fourier Transforms. Addison-Wesley, Reading (1965)

9. Zhao, H., Ma, W.: Mixed lump-kink solutions to the KP equation. Comput. Math. Appl. 74(6), 1399-1405 (2017)

10. Zhang, J., Ma, W.: Mixed lump-kink solutions to the BKP equation. Comput. Math. Appl. 74(3), 591-596 (2017)

11. Jiang, C., Zhang, F., Li, T.: Synchronization and antisynchronization of N-coupled fractional-order complex chaotic systems with ring connection. Math. Methods Appl. Sci. 41(7), 2625-2638 (2018)

12. Zhang, X., Liu, L., Wu, Y., Cui, Y.: Existence of infinitely solutions for a modified nonlinear Schrödinger equation via dual approach. Electron. J. Differ. Equ. 2018, 147 (2018)

13. Zhang, X., Jiang, J., Wu, Y., Cui, Y.: Existence and asymptotic properties of solutions for a nonlinear Schrödinger elliptic equation from geophysical fluid flows. Appl. Math. Lett. 90, 229-237 (2019)

14. Gasiorowicz, S: Elementary Particle Physics. Wiley, New York (1966)

15. Gelfand, I., Shilov, G.: Generalized Functions, vol. 1. Academic Press, New York (1964)

16. Passare, M.: Residues, currents, and their relation to ideals of holomorphic functions. Math. Scand. 62, 75-152 (1988)

17. Bliedtner, J., Hansen, W.: Potential Theory. An Analytic and Probabilistic Approach to Balayage. Springer, Berlin (1986)

18. Dong, X., Bai, Z., Zhang, S.: Positive solutions to boundary value problems of $p$-Laplacian with fractional derivative. Bound. Value Probl. 2017, Article ID 5 (2017)

19. Liu, L., Deng, F., Hou, T.: Almost sure exponential stability of implicit numerical solution for stochastic functional differential equation with extended polynomial growth condition. Appl. Math. Comput. 330, 201-212 (2018)

20. Andersson, M., Carlsson, H.: HP-estimates of holomorphic division formulas. Pac. J. Math. 173, 307-335 (1996)

21. Antosik, P., Mikusinski, J., Sikorski, R.: Theory of Distributions the Sequential Approach. PWN, Warsaw (1973)

22. Ikegami, T.: Compactifications of Martin type of harmonic spaces. Osaka J. Math. 23, 653-680 (1986) 
23. Shang, S., Bai, Z., Tian, Y., Yue, Y.: Periodic solution for second-order impulsive differential inclusions with relativistic operator. Bound. Value Probl. 2018, Article ID 173 (2018)

24. Chen, S., Ma, W.: Lump solutions to a generalized Bogoyavlensky-Konopelchenko equation. Front. Math. China 13(3), 525-534 (2018)

25. Zhang, X., Wu, Y., Cui, Y.: Existence and nonexistence of blow-up solutions for a Schrödinger equation involving a nonlinear operator. Appl. Math. Lett. 82, 85-91 (2018)

26. Meng, B., Wang, X.: Adaptive synchronization for uncertain delayed fractional-order Hopfield neural networks via fractional-order sliding mode control. Math. Probl. Eng. 2018, Article ID 1603629 (2018)

27. Noor, M.: Mixed variational-like inequalities. Commun. Appl. Nonlinear Anal. 1, 63-75 (1994)

28. Wu, J., Zhang, X., Liu, L., Wu, Y., Cui, Y.: Convergence analysis of iterative scheme and error estimation of positive solution for a fractional differential equation. Math. Model. Anal. 23(4), 611-626 (2018)

29. Yang, J., Ma, W., Qin, Z.: Lump and lump-soliton solutions to the (2+1)-dimensional Ito equation. Anal. Math. Phys. 8(3), 427-436 (2018)

Submit your manuscript to a SpringerOpen ${ }^{\circ}$ journal and benefit from:

- Convenient online submission

Rigorous peer review

Open access: articles freely available online

High visibility within the field

Retaining the copyright to your article

Submit your next manuscript at $\boldsymbol{\triangleright}$ springeropen.com 